\title{
MedienPädagogik
}

Zeitschrift für Theorie und Praxis der Medienbildung

\section{Die Auswirkungen medialer Angebote auf das Körperbild von Jugendlichen}

\author{
Eine experimentelle Studie mit impliziten und expliziten Methoden
}

Natalie Baumgartner-Hirscher und Jörg Zumbach

\begin{abstract}
Zusammenfassung
Der erhöhte Medieneinfluss trägt massgeblich zur Verbreitung eines aktuellen Frauenbildes bei, welches den Rezipientinnen und Rezipienten ein unrealistisches und ungesundes Bild des weiblichen und männlichen Körpers vermittelt (Dolan 1996; Stahr, Barb-Priebe, und Schulz 2003). Dieser soziokulturelle Einfluss ist neben biologischen, familiären und individuellen Faktoren einer der Entstehungsbedingungen für Körperbildstörungen, die ggf. auch zu Essstörungen führen können. Das Ziel der vorliegenden Studie ist es, die Frage zu beantworten, wie sich bestimmte mediale Angebote auf die Entwicklung des Körperbildes bei Jugendlichen auswirken, da diese als besonders anfällig für die Verinnerlichung und Übertragung von Medieninhalten gelten. Medien übernehmen beim Aufwachsen von Kindern und Jugendlichen zentrale Aufgaben. Sie beeinflussen das Handeln, die Einstellungen und das Wissen der Kinder (Schorb 2005). Die vorliegende Studie untersucht mit Hilfe des Implicit Association Tests (Greenwald, McGhee, und Schwartz 1998) und expliziten standardisierten Fragebögen sowie Körperbildskalen die Reaktion von Schülerinnen und Schüler auf idealisiertes Stimulusmaterial und versucht, den Zusammenhang von häufigem Medienkonsum und Diskrepanzen in der Wahrnehmung des Körperbildes zu ermitteln. Es konnten Auswirkungen des medialen Konsums attraktiver Darstellerinnen und Darsteller auf impliziter Ebene festgestellt werden. Auf expliziter Ebene, sowie bezüglich der Darstellung von Körperidealen, konnten keine Effekte gefunden werden.
\end{abstract}

The effect of medial offers on the body image of adolescents - A experimental study with implicit and explicit methods

\begin{abstract}
The mass media shows and promotes the actual standards of women and men's appearance and bodies. These means a thin body ideal for women and an over musculine ideal for men which is mostly achieved with unhealthy methods (Dolan 1996; Stahr, BarbPriebe, and Schulz; 2003). This sociocultural influence is besides biological, family and other individual factors essential for the development of body image disorders and as a consequence for eating disorders. Mass Media influence the Growing-up of Children,
\end{abstract}


especially their actions, knowledge and attitudes (cf. Schorb 2005). The aim of this work is to answer the question of how the media impact works on the development of the body image of adolescents. This study investigate the reaction of students on stimuli in terms of fotos and videos presenting the actual beauty ideal with the help of standardized questionnaires, Figure Rating Scale (Stunkard, Sorensen, and Schulsinger 1983) and the Implicit Association Test (Greenwald, McGhee, and Schwartz 1998) and try to find discrepancies between media consumption and negative impacts on individual body images. Effects of the reception of attractive media actors could be found with implicit methods. With explicit methods and with regard to the presentation of the thin media ideal could not be found any significant influence.

\section{Einleitung}

Essstörungen gehören zu den häufigsten psychosomatischen Störungen im Kindesund Jugendalter (Hölling und Schlack 2007). Die Mortalitätsrate liegt bei bis zu $6 \%$ und längerfristig überwindet nur jede beziehungsweise jeder zweite Erkrankte seine Sucht (Gerlinghoff und Backmund 2004). Es werden eine Vielzahl an Faktoren diskutiert, die sich auf die Entwicklung verschiedenster Essstörungen auswirken (Cuntz und Hillert 2008; Fichter 2013; Stahr, Barb-Priebe und Schulz 2003). Neben biologischen, individuellen und familiären Faktoren werden auch soziokulturelle Faktoren erwogen (Gerlinghoff und Backmund 2004). Zu diesen soziokulturellen Faktoren gehören unter anderem der von Frauen wahrgenommene gesellschaftliche Druck schlank zu sein, das medial präsentierte Schönheitsideal und das veränderte Rollenbild der Frau (vgl. Fichter 2008; Garner 1991; Geissner und Schary 2005; Jacobi, Paul, und Thiel 2004; Jäger 2005).

\section{Theoretischer Hintergrund}

Die Auswirkungen eines verstärkten Medienkonsums mit der einhergehenden Vermittlung eines attraktiven medialen Schönheitsideals zählen zu den Risikofaktoren zur Entwicklung eines gestörten Körperbildes und des Weiteren zu Essstörungen (vgl. Hawkins, Richards, Granley, und Stein 2004; Stice, Shipak-Neuberg, Shaw, und Stein 1994). Aktuelle Forschungen versuchen den Zusammenhang zwischen dem Medienkonsum und Körperunzufriedenheit auf Basis der sozialkognitiven Lerntheorie (Bandura 1971) und der Theorie sozialer Vergleichsprozesse (Festinger 1954) zu erklären. Demnach kann die Rezeption attraktiver Medienhandelnder zu Vergleichs- und Imitationsprozessen durch die Zusehenden führen (Engeln-Maddox 2005; Hargreaves und Tiggemann 2003; Leffelsend, Mauch, und Hannover 2004). 
Die sozialkognitive Lerntheorie (Bandura 1971) geht davon aus, dass Menschen durch Beobachtung des Verhaltens anderer Personen lernen. Das gewünschte Verhalten kann entweder bereits verinnerlicht sein und durch die Beobachtung anderer Personen aufgerufen werden oder es kann auch vollständig nachgeahmt werden. Voraussetzung für die Nachahmung sind die Einschätzungen an das Ergebnis des Nachahmungsprozesses und die Bewältigbarkeit durch den Lernenden (Jonas und Brömer 2002). Rezipientinnen und Rezipienten ahmen Vorbilder aus den Medien demzufolge nach, wenn sie davon ausgehen, dass es für sie einen Vorteil bringt. Die Schönheitsindustrie und auch Casting- beziehungsweise Realityshows tragen dazu bei, dass vermittelt wird, dass man dem attraktiven Medienschönheitsideal entsprechen kann, was den sozialen Vergleichsprozess unterstützen beziehungsweise auslösen kann (vgl. Van Bebber 2013).

Die Selbstevaluierung der Rezipierenden kann über die Theorie des sozialen Vergleichs (Festinger 1954) erklärt werden. Personen vergleichen sich - unter anderem unter Einflussnahme bestimmter Faktoren der Persönlichkeit - mit Medienpersönlichkeiten. Bezüglich der Attraktivität kann es hier oftmals zu aufwärtsgerichteten Vergleichen kommen, das heisst, dass die Medienakteurinnen und Medienakteure als attraktiver wahrgenommen werden und die Zusehenden sich infolgedessen schlechter bewerten. Dadurch können Unzufriedenheiten bis hin zu depressiven Stimmungslagen entstehen (Leffelsend et al. 2004; Pinhas, Toner, Ali, Garfinkel, und Stuckless 1999; Stice und Bearman 2001).

In Zusammenhang mit der Theorie des sozialen Vergleichs bestätigt die Theorie der Kontrasteffekte die schlechtere Beurteilung des eigenen Körpers nach der Rezeption des medialen Attraktivitäts- oder Körperideals. Die Bewertung eines Objekts, dem ein negativ bewertetes vorangeht, fällt besser aus, als wenn diesem ein positiv bewertetes vorangeht (Schemer 2003; Thornton und Moore 1993).

Insbesondere das Medium Fernsehen vermittelt laut der Theorie des Kultivationsansatzes (Gerbner, Gross, Morgan, und Signorielli 1986) Verhaltensmuster und Meinungen, die von den Rezipierenden im Sinne einer Rekonstruktion der Realität wahrgenommen werden. Je höher der Fernsehkonsum einer Person ist, desto mehr werden medial vermittelte Meinungen angenommen (Schramm und Hasebrink 2004).

Der Mere Exposure Effekt (Zajonc 1968) verstärkt diesen Ansatz dahingehend, dass Personen eher dazu neigen einen Reiz zu mögen, je mehr sie diesem Reiz ausgesetzt sind. Mehrfache Darbietung führt zu einer Art Familiarität und diese zwangsläufig zu einer positiveren Einstellung. Die permanente Präsentation attraktiver Medienpersönlichkeiten führt zu einer positiveren Einstellung und macht eine Imitation dieser für die Zusehenden erstrebenswerter (vgl. Holmstrom 2004). 


\section{Kinder und Medien}

Kinder wachsen heute nicht mehr wie früher vorwiegend in ihrem Familienverband, pädagogischen Institutionen und ihrem Freundeskreis auf. Paus-Hasebrink und Bichler (2008) sehen die Mediensozialisation von Kindern und Jugendlichen als wichtigen Punkt in ihrer Entwicklung und als massgebend für den weiteren Umgang mit medialen Angeboten im Erwachsenenalter. Medien übernehmen beim Aufwachsen von Kindern und Jugendlichen zentrale Aufgaben. Sie beeinflussen das Handeln, die Einstellungen und das Wissen der Kinder (vgl. Schorb 2005). Die heutige Kindheit beinhaltet nicht mehr ausschliesslich Erfahrungen primärer Natur, sondern wird in vielen Lebenslagen und Settings von den Massenmedien begleitet beziehungsweise beeinflusst. Kinder und Jugendliche sind in ihrer Identitätsentwicklung im Allgemeinen wesentlich leichter zu beeinflussen als Erwachsene (Treumann et al. 2007; Wegener 2008). Durch die fortschreitende Mediatisierung kommt es zu Veränderungen zwischen den Generationen und Sozialisationsagenturen. Kinder lösen sich aus alten Abhängigkeiten und werden stattdessen medial «aufgefangen» und an den Markt gebunden. Medien dienen hier verstärkt zur Selbst- und Fremderfahrung. Rollenbilder und Körperbilder werden durch Medienheldinnen und Medienhelden vermittelt und dienen den Kindern und Jugendlichen oft als erste Instanz der Orientierung (PausHasebrink und Bichler 2008).

Vocks und Legenbauer (2010) beschreiben ein multifaktorielles Modell zur Entstehung und Aufrechterhaltung von Störungen des Körperbildes. Neben individuellen Faktoren beeinflussen auch soziokulturelle Faktoren die Entwicklung des Körperbildes. Mädchen entwickeln immer früher eine verzerrte Körperwahrnehmung und verinnerlichen den Wunsch nach einem idealen Körper (Hölling und Schlack 2007). Diese veränderte Körperwahrnehmung gilt als ein Indikator für die Entwicklung von Essstörungen (Brunner und Resch 2015). Ansätze zur Prävention von Essstörungen erscheinen so bereits im Grundschulalter und in der Sekundarstufe I als sinnvoll (vgl. Berger 2006). Durch eine Behandlung des Themas im Unterricht dieser Altersstufe kann flächendeckend erreicht werden, dass Kinder und Jugendliche über die Wirkmechanismen der Massenmedien zumindest aufgeklärt beziehungsweise aufmerksam gemacht werden.

Aktuelle pädagogische Projekte nennen als Inhalt das Bewusstmachen des Einflusses durch medial vermittelte Ideale und Hinterfragen diese aktiv (vgl. Berger 2006; Brokemper 2009; Dannigkeit Köster und Tuschen-Caffier 2007; Dennhardt 2008; Schlevogt 2002; Smolak, Levine, und Schermer 1998). Inhalte derzeitiger pädagogischer Projekte sind die Vermittlung von Life-Skills, die kritische Diskussion des medialen Schönheitsideals, das Hinterfragen von Rollenbildern und der eigenen Sexualität, sowie das Stärken der Genussfähigkeit zum Beispiel in Form von Körperübungen (vgl. Gille 2014; Niedballa 2001; Schlevogt 2002). 


\section{Der Begriff «Schönheit»}

Aktuelle Studienergebnisse zeigen eine Vielzahl von Faktoren, die für die Beurteilung der Attraktivität massgeblich sind. Bezüglich der Gesichtsattraktivität können in der Literatur immer wiederkehrende Merkmale genannt werden, bei denen ein Konsens herrscht. Aufbauend auf dem Multiple Fitness Model (Cunningham, Roberts, Barbee, Druen, und Wu 1995; Cunningham; Barbee, Philhower, Rhodes, und Zebrowitz 2002) sind bei Frauen Merkmale des Kindchenschemas (Lorenz 1943; Aronson, Wilson, und Akert 2009), sexuelle Reifezeichen sowie Merkmale, welche die Jugendlichkeit eines Gesichts anzeigen, Faktoren, die die Beurteilung der Attraktivität massgeblich mitbestimmen. Die Beeinflussung der Attraktivitätsbeurteilung anhand von Zeichen der Symmetrie gilt inzwischen als überholt. Zwar zeigen Studien, dass die Symmetrie in Gesichtern durchaus positiv wirken kann. Jedoch gelten leichte Abweichungen, wie zum Beispiel Muttermale oder Schönheitsflecke als besonders attraktiv (Schuster 1993; Hönn und Göz 2007). Neueste Studien arbeiten mit der Technik des Morphings. Durch Überlagerungen sogenannter Durchschnittsgesichter entstehen Gesichter, die attraktiver als die ursprünglichen Gesichter wirken (Braun, Gründl, Marberger, und Scherber 2001; Langlois und Roggmann 1990). Faktoren männlicher Attraktivität sind deutlich weniger manifestiert und unterliegen einer Schwankungsbreite. Es wird vermutet, dass diese Schwankungen in Zusammenhang mit dem hormonellen Zyklus der Frau stehen könnten (Klusmann und Berner 2011).

\section{Aktueller Forschungsstand}

Der aktuelle Forschungsstand belegt den Einfluss der Medien auf das Körperbild von Kindern, Jugendlichen und Erwachsenen. Es ist jedoch festzustellen, dass die Wirkung der Medien als alleiniger Einflussfaktor auf das Körperbild und weiterführend auf die Entwicklung einer Essstörung umstritten ist (vgl. Dittmar 2005; Schemer 2007; Šmahel et al. 2018, Stice et al. 1994). Faktoren wie der Peer-Einfluss und der Einfluss der Familie übernehmen neben individuellen Eigenschaften eine moderierende Wirkung (Dolan 1996). Baumann (2009) kritisiert am derzeitigen Forschungsstand, dass derartige individuelle Einstellungen und subjektive Körpereinschätzungen der Versuchspersonen grossteils nicht abgefragt werden. Ein weiterer kritisch zu betrachtender Punkt ist, dass in der Versuchsdurchführung die schlanken Körper der Models und Medienprotagonistinnen und Medienprotagonisten mit einem ebenso attraktiven Gesicht verbunden waren. Das Konstrukt der Attraktivität wurde so nicht klar festgelegt. Ferner weist ein Grossteil der Studien keine Kontrollgruppen beziehungsweise keinen Einsatz vergleichenden Stimulusmaterials in Form von nichtkörperbezogenen Bildern oder Darstellungen fülligerer Körper auf (vgl. Baumann 2009; Holmstrom 2004). Weiters ist auffällig ist, dass in vielen Studien fast ausschliesslich Stimumulusmaterial in Form von Frauenkörpern und Frauengesichtern vorgelegt 
wurde und dieses auch meist von weiblichen Versuchspersonen beurteilt wurden (vgl. Grabe, Ward, und Hyde 2008; Posavac, Posavac, und Weigel 2001; Slater und Tiggemann 2006; Yamamiya, Cash, Melnyk, Posavac, und Posavac 2005). Generell ist anzumerken, dass die Ergebnisse dieser quantitativen und qualitativen Studien auf expliziten Methoden in Form von Fragebögen und Körperbildeinschätzungen basieren (vgl. Heinberg und Thompson 1995; McCabe und Ricciardelli 2001; 2005). Eine Erweiterung des Forschungsstandes durch implizite Methoden ist Ziel der vorliegenden Studie. Es wird im Folgenden ein implizites Messinstrument konzipiert. Explizit erhobene Daten können so mit implizit erhobenen Daten verglichen werden. Aktuell werden moderierende Faktoren, die Einfluss auf die Rezeption und Medienwirkung des Schönheitsideals auf das individuelle Körperbild suggerieren, diskutiert. Eine weitere Schwachstelle in aktuellen Studien ist, dass moderierende Faktoren im Versuchsdesign oft nicht berücksichtigt werden.

In der vorliegenden Studie wird dazu neben dem Selbstbewusstsein auch die Zufriedenheit mit dem eigenen Körper, die Einschätzung der eigenen Silhouette und Silhouetten von Wunschkörpern beider Geschlechter abgefragt. In einer weiteren Studie wird zudem eine Separierung der Darstellung von dem Medienideal entsprechenden Körperdarstellungen ohne dazugehörige Gesichter versucht (vgl. Baumgartner-Hirscher 2017).

\section{Fragestellung und Hypothesen}

Das Methodenrepertoire der aufgezählten Studien beinhaltet ausschliesslich explizite Methoden in Form von Fragebögen. Hier besteht die Gefahr durch die Versuchspersonen, persönliche Inhalte nicht preisgeben zu wollen, beziehungsweise nach sozialer Erwünschtheit zu antworten. Um die Auswirkungen des Konsums medialer Angebote mit darin vermittelten Schönheitsnormen zu untersuchen, wird in der folgenden Studie neben expliziten Methoden auch eine implizite gewählt. Die Datenerhebung der folgenden Studie erfolgt mittels eines Implicit Association Tests (Greenwald, McGhee, und Schwartz 1998). Der Implicit Association Test (IAT) ist ein Instrument, das vorwiegend zur Messung von Einstellungen und Stereotypen verwendet wird. Es wird die Assoziationsstärke zwischen zwei Konzepten des Gedächtnisses durch die Messung von Reaktionszeiten erhoben. Nach Auswahl geeigneter Stimuli und Konzeption des IATs versucht die vorliegende Studie, die Antwort auf die Frage der Beeinflussung von medialen Inhalten auf das eigene Körperbild zu beantworten. Überprüft werden auch Zusammenhänge zwischen häufigem Medienkonsum und Einschätzungen des Körperbildes beziehungsweise dem Wunsch nach dem Idealkörper. Es wird die Vermutung aufgestellt, dass Auswirkungen des Medienkonsums mit impliziten Methoden präziser gemessen werden können, da diese weniger manipulierbar durch die Versuchspersonen sind. Aktuelle Forschungsergebnisse weisen vermehrt darauf hin, 
dass auch ein Geschlechterunterschied in der Medienrezeption und Wirkung besteht. Mädchen leiden demnach stärker unter den Auswirkungen medial verbreiteter Ideale (u.a. Cusumano und Thompson 2001; Hargreaves und Tiggemann 2004). Auch diese Hypothese wird untersucht.

\section{Stichprobe und Design}

Die Studie fand im Rahmen einer Projektwoche an einem Salzburger Gymnasium in den zehnten bis zwölften Schulstufen sowie am Versuchslabor der naturwissenschaftlichen Fakultät der Universität Salzburg statt. Es nahmen 108 Versuchspersonen im Alter zwischen 15 und 32 teil $(M=17.35$; $S D=2.76)$, davon waren 40 weiblich und 68 männlich.

\section{Material}

Untersucht wurde der Einfluss der Intervention auf zwei explizite und ein implizites Mass zur Erfassung des Körperbildes bzw. Körperselbstkonzepts. Die explizite Masse wurde mit den Frankfurter Körperkonzeptskalen (Deusinger 1998), der Figure Rating Scale (Stunkard, Sorensen, und Schulsinger 1983) und einem selbst konzipierten Fragebogen zum Medienkonsumverhalten erfasst. Das implizite Körperselbstkonzept wurde mit einem Impliziten Assoziationstest (IAT) erfasst.

\section{Frankfurter Körperkonzeptskalen}

Zur Feststellung der Körperzufriedenheit wurden die Frankfurter Körperkonzeptskalen (FKKS) herangezogen; insgesamt lagen dabei drei Messzeitpunkte vor. Die Frankfurter Körperkonzeptskalen dienen der Erfassung und Bestimmung des Körperbildes und Selbstkonzeptes der jeweiligen Versuchsperson. Die neun Subskalen des Fragebogens erfassen ein System von Einstellungen zum eigenen Körper. Insgesamt wurden aus 64 vorhandenen Items in neun Subskalen 54 Items aus sieben Subskalen herausgenommen. Die Subskalen, welche die Einstellungen zur Sexualität und dissimilatorischen Körpervorgängen abfragen, wurden ausgeschlossen. Die verwendeten sieben Subskalen der Frankfurter Körperkonzeptskalen erfassen die Körperkonzepte «Gesundheit und körperliches Befinden» (Cronbach's $\alpha=$ Vortest: 0.66/ Nachtest: $0.76 /$ verzögerter Nachtest: 0.75$)$, «Pflege des Körpers» ( $\alpha=0.75 / 0.79 / 0.74)$, «Körperliche Effizienz» ( $\alpha=0.84 / 0.85 / 0.88)$, «Körperkontakt» ( $\alpha=0.79 / 0.83 / 0.86)$, «Selbstakzeptanz des Körpers» ( $\alpha=0.80 / 0.83 / 0.78$ ) und «Äussere Erscheinung» ( $\alpha$ $=0.71 / 0.71 / 0.79)$. Die Aussagen wurden auf einer sechsstufigen Likert-Skala von 1 («trifft gar nicht zu») bis 6 («trifft sehr zu») beurteilt. Zusammengefasst lagen die internen Konsistenzen der verwendeten Subskalen der Frankfurter Körperkonzeptskalen zwischen $\alpha=0.66$ und $\alpha=0.86$. 


\section{Figure Rating Scale}

Die Figure Rating Scale dient als Instrument zur Erfassung der Körperunzufriedenheit und des subjektiven Körperbildes bei Männern und Frauen (Stunkard et al. 1983). Die Skala umfasst jeweils neun gezeichnete Silhouetten von Frauen- und Männerkörpern und wurde zu zwei Messzeitpunkten vorgelegt. Die Probanden sollten angeben, welche Silhouetten von anderen am attraktivsten bewertet werden, welche Silhouette ihnen selbst am besten gefällt und welche Silhouette die eigene Körperform am besten abbildet. Die internen Konsistenzen der Figure Rating Scale liegen - nach Ausschluss des ersten Items - im Vortest und im Nachtest bei Cronbach's $\alpha=0.78$.

\section{Medienfragebogen}

Im Nachtest wurden die Versuchspersonen zu ihrem Medienkonsumverhalten befragt. Der Fragebogen bestand aus zehn Items und erfasste die Konsumdauer verschiedener medialer Angebote sowie persönlich wahrgenommenen Effekte und Einstellungen zur Nutzung von Printmedien, Internet, Radio und Fernsehen.

\section{Impliziter Assoziationstest}

Der Implicit Association Test ist ein computerbasiertes Reaktionszeitmessverfahren, das die Stärke der Assoziationen zwischen zwei Elementen des Gedächtnisses erhebt (Greenwald, McGhee, und Schwartz 1998). Das Messverfahren basiert auf der Grundannahme, dass sich Kategorien, welche miteinander assoziiert werden, schneller einander zuordnen lassen als welche, die die Versuchsperson nicht miteinander in Verbindung stellt. Anhand der Messung dieser Reaktionszeiten wird der sogenannte IAT-Effekt ermittelt, welcher die Differenzen der Reaktionszeiten angibt. Das Messverfahren wird vorwiegend zur impliziten Messung von Einstellungen gegenüber Objekten des Selbstwerts und Selbstbildes, sowie von Identitäten und Stereotypen verwendet. Generell gilt der IAT als valide und weist hohe interne Konsistenzen auf (Greenwald et al. 1998; Greenwald, PoehIman, Uhlmann, und Banaji 2009). Laut Greenwald et al. (1998) besteht der Vorteil der impliziten Messung (insbesondere bei Konzepten des Selbstwertes und Selbstbildes) darin, dass Antwortsequenzen im Sinne der sozialen Erwünschtheit besser vermieden werden können (Banaji 2001; Greenwald et al. 1998). Des Weiteren können Einstellungen erhoben werden, die der Versuchsperson möglicherweise selbst nicht bewusst sind. Der IAT wird als Einzeltestverfahren am Computer bearbeitet. Der Test besteht aus insgesamt fünf Durchgängen und dauert durchschnittlich fünf bis sieben Minuten. In jedem Durchgang ordnen die Versuchspersonen mittels Tastendruck Reizwörter und Reizbilder einem von zwei Zielkonzepten zu. Die Konzeption eines passenden IATs bedarf im Vorfeld der Auswahl des geeigneten Stimulusmaterials. Um möglichst geeignete Wörter und 
Bilder auszusuchen, wurde hier entsprechend eine Vorstudie durchgeführt. Dies sollte gewährleisten, dass die Stimuli im IAT von den Versuchspersonen eindeutig kategorisiert werden können. Hierzu wurden den Versuchspersonen Bilder und Wörter gezeigt, welche diese beurteilen mussten. So mussten hier aus 105 Gesichtsbildern und 30 Wörtern die geeignetsten ermittelt werden. Anhand einer Stichprobe aus 84 Schülerinnen und Schüler im Alter von 15 bis 20 Jahren $(M=16.61 ; S D=1.2 ; 42$ weiblich, 42 männlich) wurden die Gesichtsfotos bewertet. Die Ermittlung der Worte erfolgte anhand von Fragebögen mit 80 Versuchspersonen mit einem Altersdurchschnitt von 16.77 (SD = 1.18; 26 weiblich, 52 männlich). Die Beurteilung der Bilder und Wörter wurde mit Hilfe eines Fragebogens mit einer fünfstufigen Likert-Skala (von 1 $=$ «Sehr zutreffend» bis $5=$ «Absolut unzutreffend») durchgeführt. Die Wörter wurden in Anlehnung an Project Implicit (implicit.harvard.edu) ausgewählt. Das Bildmaterial stammt aus der wissenschaftlichen Fotodatenbank IAPS (International Affectiv Picture System von Lang, Bradley, und Cuthbert 1999), Fotos einer Modelagentur sowie privat zur Verfügung gestellten Fotos. Die Fotos wurden mit Adobe Photoshop CC 2014 seriell modelliert, d.h. aus einem Foto wurden durch geringfügige Veränderungen am Gesicht eine komplette Serie aus 10 Fotos hergestellt (siehe Abbildung 1). Um die Bildserien herzustellen, wurde bei den Gesichtsfotos an den Augen (Grösse, Stellung und Form), an der Form des Gesichts und der Symmetrie, an der Nase, am Mund (Form und Fülle der Lippen) und an den Haaren (Ansatz und Fülle) sowie bezüglich der Retusche von Unebenheiten und Falten manipuliert.
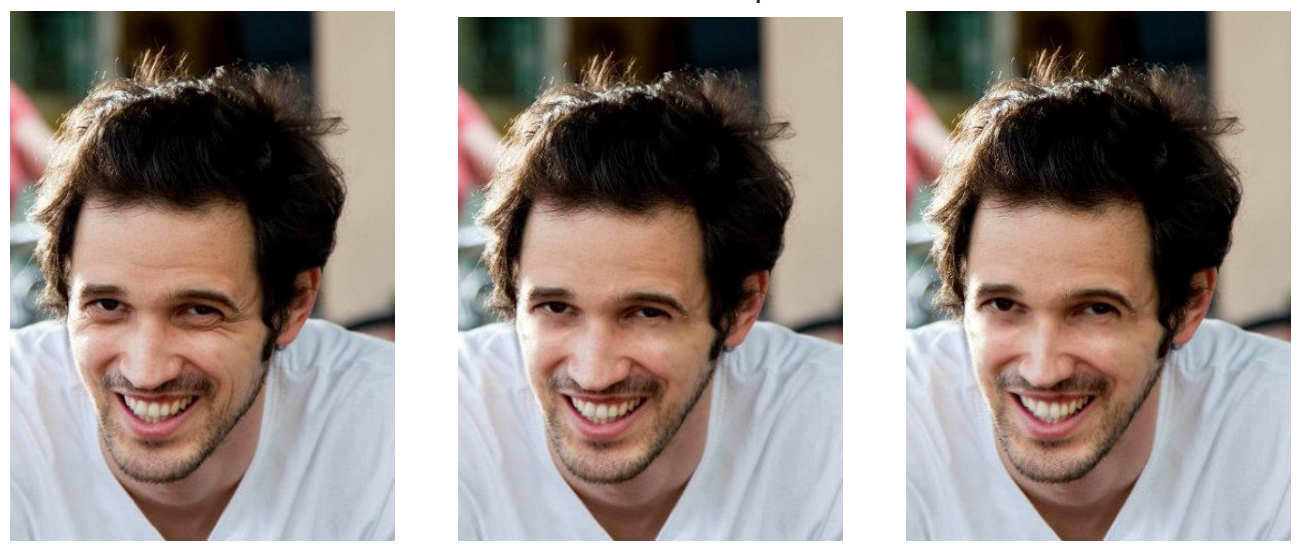

Abb. 1.: Manipuliertes Stimulusmaterial für den IAT.

Mittels einer Faktorenanalyse und dem Vergleich der Mittelwerte wurden die am besten und schlechtesten wahrgenommenen Wörter sowie die attraktivsten und unattraktivsten Bilder ermittelt und als Stimulusmaterial in den IAT übernommen ( $\alpha=$ 0.78). 


\section{Durchführung}

Die Klassen und Studierendengruppen wurden per Zufallsprinzip drei Versuchsgruppen zugeteilt. Die Versuchspersonen der ersten Versuchsgruppe bekamen zeitgleich Videosequenzen aus zwei US-Amerikanischen TV-Serien gezeigt, in denen westliche Schönheits- und Schlankheitsideals dargeboten werden, d.h. dass bei den Protagonistinnen und Protagonisten der Serien BMI-Werte unter 19 vorliegen. Der zweiten Versuchsgruppe wurde eine Präsentation mit Fotos von weiblichen und männlichen Models und Schauspielern gezeigt. Sowohl die Fotos der Versuchsgruppe wie auch der Kontrollgruppe enthielten keinen Text oder Produktwerbungen, die vom Inhalt hätten ablenken können.

Vor und nach den Interventionen wurde der Implicit Association Test durchgeführt und die Fragebögen ausgefüllt. Zum Abschluss wurde mit einem einseitigen Fragebogen der Medienkonsum der Versuchspersonen erhoben (einen detaillierten Einblick gibt auch Baumgartner-Hirscher 2017).
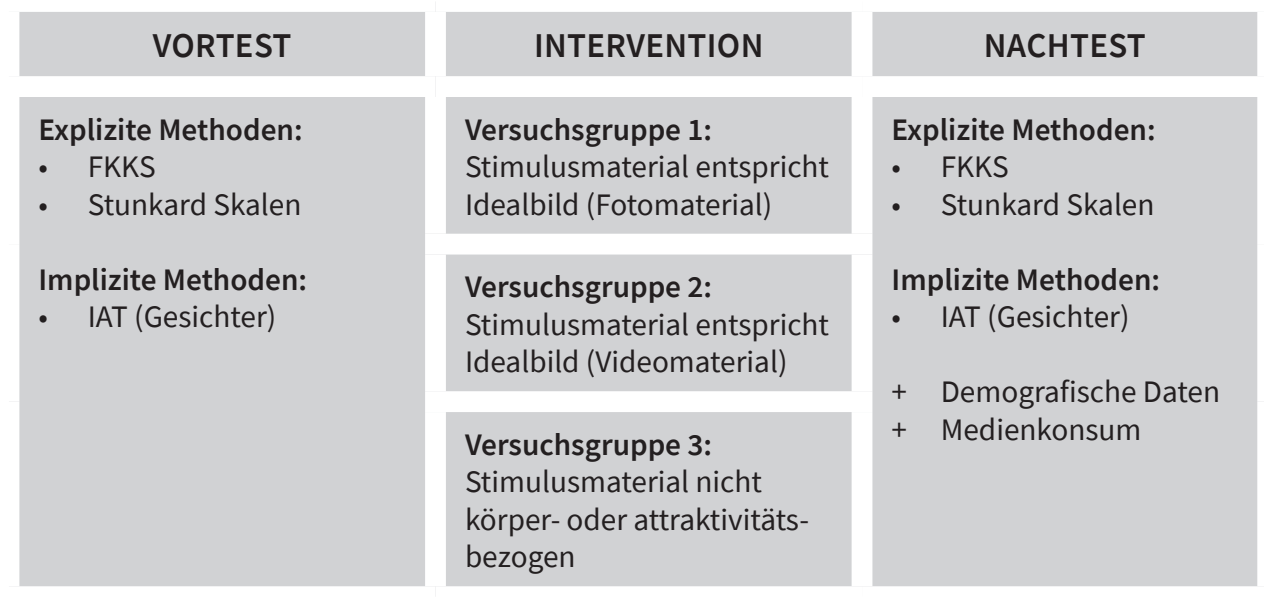

Tab. 1.: Design und Ablauf der Studie.

\section{Durchführung IAT}

Der eigens konzipierte IAT wurde den Versuchspersonen am Computer zur Verfügung gestellt. In der ersten Phase sollten die Versuchspersonen Fotos von Frauen und Männern der Kategorie «attraktiv» oder «unattraktiv» zuordnen. In der zweiten Phase wurden den Versuchspersonen Worte gezeigt, welche sie der Kategorie «gut» oder «schlecht» zuordnen. In der dritten Phase werden nun die ersten beiden Phasen zusammengefügt. Auf einer Antworttaste befanden sich die Kategorien «attraktiv» und «gut», auf der anderen die Kategorien «unattraktiv» und «schlecht» (siehe Abbildung 2). 


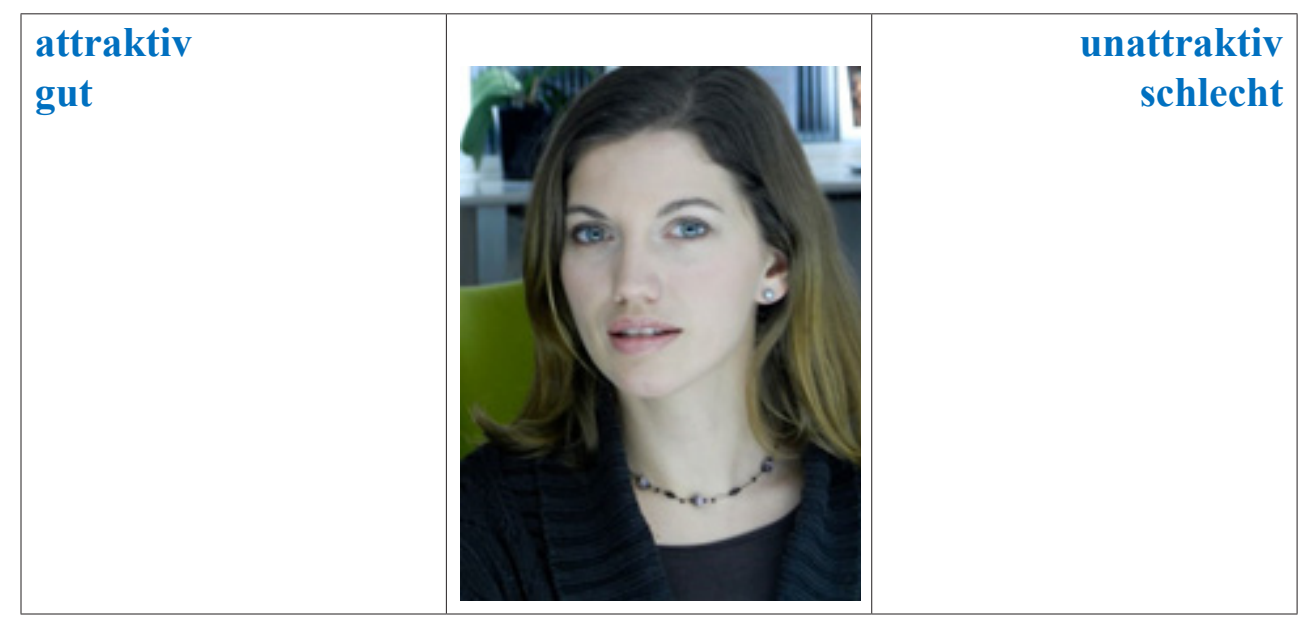

Abb. 2.: Beispielbild IAT - Phase 3.

In der vierten Phase wurden die Antworttasten vertauscht. In der fünften Phase waren die Antworttasten nochmals doppelt belegt. Hier befanden sich auf einer Seite die Kategorien «attraktiv» und «schlecht», auf der anderen die Kategorien «unattraktiv» und «gut».

\section{Ergebnisse der expliziten Erhebung}

Zur Untersuchung von Effekten des Treatments auf die abhängigen Variablen wurde im Bereich der expliziten Messwerte eine Kovarianzanalyse berechnet. Hier wurden die Werte der einzelnen Skalen der FKKS aus dem Vortest als Kovariaten herangezogen, die Ergebnisse aus dem Nachtest dieser Skalen als abhängige Variablen. Zusätzlich wurde der BMI der Versuchspersonen als weitere Kovariate in das Design integriert.

Dabei zeigte sich kein signifikanter Haupteffekt, welcher auf das Treatment hätte zurückgeführt werden können $\left(F(12,112)=0,83 ; p=0.623 ; \eta^{2}=0.08\right)$. Eine genauere Betrachtung der Mittelwerte (siehe Tabelle 2) zeigt im Nachtest einen höheren Wert bei der Skala «Pflege des Körpers» bei der Video-Bedingung gegenüber der Kontrollgruppe, welche wiederum leicht höher lag als die Gruppe mit der Bildbetrachtung. Auch bei der Skala «Körperliche Effizienz» und der Skala «Gesundheit und körperliches Befinden» zeigen sich im Nachtest in der Videogruppe leicht höhere Werte als in der Kontrollgruppe und dort wiederum höhere Werte als in der Bildgruppe. In der Subskala «Körperkontakt» sowie in der Subskala «Selbstakzeptanz des Körper» weisen die Videogruppe sowie die Bildgruppe höhere Werte auf als die Kontrollgruppe. In der Skala «Äussere Erscheinung» liegen die Mittelwerte in der Bildgruppe leicht über den Werten in der Videogruppe und deutlich über den Werten in der Kontrollgruppe. 


\begin{tabular}{|l|l|c|r|}
\hline Skalen & Versuchsgruppe & Mittelwert & SD \\
\hline «Pflege des Körpers» & Bildbedingung & 2.36 & 0.76 \\
& Kontrollgruppe & 2.65 & 0.81 \\
& Videobedingung & 3.01 & 0.57 \\
& Gesamt & 2.60 & 0.77 \\
\hline «Körperliche Effizienz» & Bildbedingung & 1.93 & 0.78 \\
im Nachtest & Kontrollgruppe & 1.97 & 0.71 \\
& Videobedingungen & 1.98 & 0.63 \\
& Gesamt & 1.95 & 0.72 \\
\hline «Körperkontakt» & Bildbedingung & 2.65 & 1.01 \\
im Nachtest & Kontrollgruppe & 2.57 & 1.03 \\
& Videobedingungen & 3.02 & 1.05 \\
& Gesamt & 2.73 & 1.03 \\
\hline «Selbstakzeptanz des Körpers» & Bildbedingung & 2.31 & 0.66 \\
im Nachtest & Kontrollgruppe & 2.25 & 0.99 \\
& Videobedingungen & 2.63 & 0.63 \\
& Gesamt & 2.38 & 0.75 \\
\hline «Gesundheit und körperliches & Bildbedingung & 1.45 & 0.59 \\
Befinden» im Nachtest & Kontrollgruppe & 1.63 & 0.82 \\
& Videobedingungen & 1.89 & 0.71 \\
& Gesamt & 1.61 & 0.70 \\
\hline «Äussere Erscheinung» & Bildbedingung & 1.94 & 0.71 \\
im Nachtest & Kontrollgruppe & 1.41 & 0.68 \\
& Videobedingungen & 1.88 & 0.75 \\
& Gesamt & 1.79 & 0.74 \\
\hline
\end{tabular}

Tab. 2.: Skalenmittelwerte im Nachtest.

Die Analyse der Kovariaten zeigt einen signifikanten Einfluss aller Variablen ausser des BMI $\left(F(6,55)=0,46 ; p=0.83 ; \eta^{2}=0.05\right)$. Bei der Skala «Pflege des Körpers» $(F(6,55)$ $\left.=67,47 ; p<0.001 ; \eta^{2}=0.88\right)$ zeigt sich, dass sich die Werte aus dem Vortest lediglich auf den jeweiligen Skalenwert im Nachtest signifikant auswirken $(F(1,60)=419,41$; $p$ $\left.<0.001 ; \eta^{2}=0.88\right)$, jedoch auf keinen anderen Wert.

Bei der Subskala «Körperliche Effizienz» zeigt sich ebenfalls, dass sich die Vortestwerte $\left(F(6,55)=94,22 ; p<0.001 ; \eta^{2}=0.91\right)$ ausschliesslich auf die Werte im Nachtest auswirken $\left(F(1,60)=514,1 ; p<0.001 ; \eta^{2}=0.9\right)$. Ebenso verhalten sich die Werte der Subskala «Körperkontakt» im Vortest $\left(F(6,55)=76,62 ; p<0.001 ; \eta^{2}=0.89\right)$ zum Nachtest $\left(F(1,60)=393,08 ; p<0.001 ; \eta^{2}=0.87\right)$ sowie der Skala «Selbstakzeptanz des Körpers» im Vortest $\left(F(6,55)=23,23 ; p<0.001 ; \eta^{2}=0.72\right)$ und im Nachtest $(F(1,60)=106,71$; $\left.p<0.001 ; \eta^{2}=0.64\right)$, und der Skala «Äussere Erscheinung» im Vortest $(F(6,55)=21,9 ; p$ $\left.<0.001 ; \eta^{2}=0.71\right)$ und im Nachtest $\left(F(1,60)=115,24 ; p<0.001 ; \eta^{2}=0.66\right)$. Die Skala «Gesundheit und körperliches Wohlbefinden» $\left(F(6,55)=17,78 ; p<0.001 ; \eta^{2}=0.66\right)$ zeigt einen Einfluss auf diese Skala im Nachtest $\left(F(1,60)=86,9 ; p<0.001 ; \eta^{2}=0.59\right)$, sowie auf die Skala «Pflege des Körpers» im Nachtest $\left(F(1,60)=514,1 ; p=0.025 ; \eta^{2}=0.08\right)$. 
Ergebnisse der Erhebung mittels der Figure Rating Scale

Zur Untersuchung von Effekten des Treatments auf die abhängigen Variablen wurde eine Varianzanalyse mit Messwertwiederholung durchgeführt. Es zeigte sich kein signifikanter Haupteffekt, der auf das Treatment zurückgeführt werden könnte $(F(2,98)$ $=0,44 ; p=0.65 ; \eta^{2}=0.01$ ). Es konnte aber ein Geschlechtereffekt festgestellt wer$\operatorname{den}\left(F(4,98)=4,30 ; p=0.003 ; \eta^{2}=0.15\right)$. Weibliche und männliche Versuchspersonen differieren in der Beurteilung der Silhouetten. Hier zeigt die genauere Betrachtung der Mittelwerte, dass Mädchen sowohl im Vortest wie auch im Nachtest bei ihrem eigenen weiblichen Wunschkörper einen geringeren Durchschnittswert angaben, wie auch bei ihrem männlichen Idealbild (siehe Tabelle 3). Generell ist hier anzumerken, dass das Idealbild des männlichen Körpers auf der Silhouettenskala deutlich über dem Wert des weiblichen Idealkörpers lokalisiert war. Den niedrigsten Wert auf der Skala erreichte die von Mädchen gewünschte weibliche Silhouette und den höchsten Wert erreichte der von Männern idealisierte Männerkörper.

\begin{tabular}{|l|l|c|r|}
\hline Silhouetten & Versuchspersonen & Mittelwert & SD \\
\hline Silhouetten & weiblich & 3.03 & 0.63 \\
(weiblich im Vortest) & männlich & 3.38 & 0.43 \\
& gesamt & 3.25 & 0.54 \\
\hline Silhouetten & weiblich & 2.96 & 0.61 \\
(weiblich im Nachtest) & männlich & 3.47 & 0.43 \\
& gesamt & 3.28 & 0.56 \\
\hline Silhouetten & weiblich & 3.67 & 0.70 \\
(männlich im Vortest) & männlich & 4.04 & 0.59 \\
& gesamt & 3.90 & 0.66 \\
\hline Silhouetten & weiblich & 3.67 & 0.66 \\
(männlich im Nachtest) & männlich & 4.00 & 0.64 \\
& gesamt & 3.87 & 0.66 \\
\hline
\end{tabular}

Tab. 3.: Vergleich der Mittelwerte nach Geschlecht. 
Ein Vergleich der Versuchsgruppen zeigt hier Unterschiede in der Beurteilung des Idealbildes, sowohl bei Frauen als auch bei Männern (siehe Tabelle 4).

\begin{tabular}{|l|l|l|c|r|}
\hline Silhouetten & \multicolumn{2}{|l|}{ Versuchspersonen } & Mittelwert & SD \\
\hline & Geschlecht & Versuchsgruppe & & \\
\hline Silhouetten & weiblich & & \\
(weiblich im & & Kontrollgruppe & 2.90 & 0.55 \\
Nachtest) & & Experimentalgruppe & 2.98 & 0.62 \\
& männlich & gesamt & 2.96 & 0.61 \\
\hline & & Kontrollgruppe & 3.49 & 0.41 \\
& & Experimentalgruppe & 3.45 & 0.44 \\
& \multirow{2}{*}{ gesamt } & gesamt & 3.47 & 0.43 \\
\hline & & Kontrollgruppe & 3.39 & 0.49 \\
& & Experimentalgruppe & 3.23 & 0.58 \\
Silhouetten & \multirow{2}{*}{ weiblich } & gesamt & 3.28 & 0.56 \\
\hline Nachntest) & & Kontrollgruppe & 3.93 & 0.60 \\
& & Experimentalgruppe & 3.63 & 0.67 \\
& \multirow{2}{*}{ männlich } & gesamt & 3.67 & 0.66 \\
\hline & & Kontrollgruppe & 4.08 & 0.65 \\
& & Experimentalgruppe & 3.95 & 0.64 \\
& gesamt & gesamt & 4.00 & 0.64 \\
\hline & & Kontrollgruppe & 4.10 & 0.63 \\
& & Experimentalgruppe & 3.80 & 0.67 \\
& & gesamt & 3.87 & 0.66 \\
\hline
\end{tabular}

Tab. 4.: Vergleich der Mittelwerte nach Treatment und Geschlecht.

Die weibliche Versuchsgruppe, die idealbesetzten Körpern in Bildern und Videomaterial ausgesetzt war (Experimentalgruppe), zeigt einen minimal höheren Wert bei der weiblichen Wunschsilhouette als die weibliche Kontrollgruppe. Die männliche Experimentalgruppe bewertet hingegen das ideale weibliche Silhouettenbild minimal geringer als die männliche Kontrollgruppe, aber deutlich höher als die weiblichen Versuchspersonen.

Auch die Bewertung der idealen männlichen Silhouette zeigt unterschiedliche Werte in den Versuchsgruppen. Mädchen aus der Experimentalgruppe bewerten nach dem intensiven Besichtigen des Bild- und Videomaterials die ideale männliche Silhouette deutlich geringer als die Kontrollgruppe. Bei den männlichen Versuchspersonen ist nur ein geringer Unterschied ersichtlich, wobei die männliche Experimentalgruppe immer noch einen geringfügig höheren Wert aufweist als die weibliche Kontrollgruppe. 


\section{Ergebnisse der impliziten Messung}

Zu Auswertung der Ergebnisse des Implicit Association Tests wurde eine separate Varianzanalyse mit Messwertwiederholung berechnet. Es konnte kein signifikanter Haupteffekt über die Zeit und allen Gruppen zusammen gezeigt werden $(F(1,89)=$ 0,$17 ; p=0.68 ; \eta^{2}=0.002$ ). Allerdings zeigte sich eine signifikante Interaktion zwischen den beiden Messzeitpunkten und der unabhängigen Variablen $(F(2,89)=3,19 ; p=0.5$; $\left.\eta^{2}=0.07\right)$. Hier zeigte eine Betrachtung der Gruppenkontraste, dass sich die Kontrollgruppe signifikant von der Gruppe mit Bildbetrachtung $(p=0.005)$ sowie der Videogruppe ( $p=0.04$ ) unterscheidet. Zwischen beiden Experimentalgruppen konnte hier kein Unterschied gezeigt werden $(p=0.63)$.

Eine genauere Betrachtung der Mittelwerte des Implicit Association Tests im Nachtest zeigt einen höheren Wert bei der Video-Bedingung gegenüber der Kontrollgruppe, welche wiederum deutlich höher lag als die Gruppe mit der Bildbetrachtung (siehe Tabelle 5).

\begin{tabular}{|l|l|c|r|}
\hline & Versuchsgruppe & Mittelwert & SD \\
\hline \multirow{2}{*}{ IAT im Nachtest } & Bildbedingung & 529.94 & 265.82 \\
& Kontrollgruppe & 441.86 & 248.37 \\
& Videobedingungen & 544.04 & 263.37 \\
& Gesamt & 507.77 & 260.91 \\
\hline
\end{tabular}

Tab. 5.: Mittelwerte des IAT im Nachtest.

Bezüglich der Frage, ob eine Korrelation zwischen überdurchschnittlich hohem Medienkonsum und Defiziten im eigenen Körperbild, welche über die Subskala «Äusseren Erscheinung» dargestellt wird, besteht. Hier konnte ein geschlechtstypischer Unterschied festgestellt werden. Bei Mädchen konnte eine Korrelation mit dem Konsum von Internet $(r=0.35 ; p<0.05)$, Zeitungen beziehungsweise Zeitschriften $(r=0.33 ; p$ $<0.05)$ beziehungsweise dem Radio $(r=0.35 ; p<0.05)$ im Vortest sowie dem Internet $(r=0.35 ; p<0.05)$ und Computerkonsum ( $r=0.33 ; p=0.05)$ im Nachtest festgestellt werden. Bei männlichen Jugendlichen konnten hingegen keine signifikanten Korrelationen festgestellt werden.

\section{Diskussion der Ergebnisse}

Vor der ersten Studie wurde in einer unabhängigen Vorstudie geeignetes Stimulusmaterial zur Konzeption des Implicit Association Tests ausgewählt. Auffällig war hierbei, dass die ausgewählten Fotos beider Kategorien, bis auf eine einzige Ausnahme, modelliert waren. Die Fotos der Kategorie «attraktiv» wurden mit Hilfe eines Weichzeichners bearbeitet (vgl. Rhodes, Yoshikawa, Clark, Lee, McKay, und Akamatsu 2001). Zusätzlich wurden die Umrisse deutlich verschmälert, die Augen und Lippen wurden vergrössert, die Nasen verkleinert und Symmetrien hergestellt (vgl. Cunningham et 
al. 1995; Rhodes et al. 2001). Die Fotos der Kategorie «unattraktiv» wurden ebenfalls bearbeitet. Die Gesichter und speziell die Nasen wurden verzerrt und deutlich grösser gestaltet. Es wurden auch asymmetrische Gesichtszüge hergestellt. Die Ergebnisse dieser Auswertung stimmen mit den gängigen Attraktivitätstheorien überein (vgl. Cunningham 1986). Durch Vergrösserung der Augen und des Mundes wurden die Gesichter dem «Kindchenschema» angepasst (vgl. Borelli und Berneburg 2010). Auch die Symmetriehypothese wurde bestätigt (vgl. Hönn und Göz 2007; Perrett et al. 1999).

Nach Auswahl der geeigneten Stimuli wurde die Hauptstudie durchgeführt. Ziel der Studie war es, die unmittelbaren Auswirkungen des Konsums medialer Bilder, die dem Schönheitsideal entsprechen, auf jugendliche Versuchspersonen zu untersuchen. Die Ergebnisse zeigen, dass es bei der expliziten Erhebung keine signifikanten Unterschiede zwischen dem Vortest und dem Nachtest gegeben hat. Die Vergleiche der Mittelwerte zeigen jedoch, dass in der Subskala «Äussere Erscheinung» die Mittelwerte der Versuchsgruppen über jenen der Kontrollgruppe lagen. Diese Subskala erfasst die Zufriedenheit der Versuchspersonen mit dem eigenen Körper. Je geringer hier die Werte waren, desto höher war die Zufriedenheit mit dem eigenen Körper und dem eigenen Aussehen. Insgesamt kann festgestellt werden, dass in allen Subskalen die Mittelwerte der Videogruppe über den Mittelwerten der Bildgruppe und der Kontrollgruppe lagen. Das heisst, dass die Körperzufriedenheit in allen Bereichen geringere Werte aufwies wie in den anderen Gruppen.

Die geringen Auswirkungen der Intervention auf das Körperbild können auf verschiedene individuelle Faktoren zurückgeführt werden (vgl. Baumann 2009; Dittmar und Howard 2004; Dittmar 2005; Schemer 2007). Zum Beispiel, weisen die Versuchspersonen eine hohe Körperzufriedenheit auf. Dieser moderierende Faktor wurde mittels der Figure Rating Scale (Stunkard et al. 1983) erhoben. Die Unterschiede zwischen den Mittelwerten bei der Beurteilung der eigenen Silhouette und der Angabe der Wunschsilhouette lagen im Zehntelbereich. Daraus kann geschlossen werden, dass die Versuchspersonen neben einer Zufriedenheit mit dem Körper kein Bestreben nach einem deutlich schlankeren Ideal haben. Dieser Faktor zeigt ein Selbstvertrauen in den eigenen Körper und gibt Anlass auszuschliessen, dass ein sozialer Vergleichsprozess stattgefunden hat (vgl. Holmstrom 2004; Schemer 2007).

Der Implicit Association Test zeigte jedoch einen signifikanten Effekt bezüglich der Versuchsgruppen. Wie angenommen, zeigten die impliziten Ergebnisse eine deutlichere Beeinflussung der Versuchspersonen als die expliziten Daten. Die Videogruppe wies eindeutig höherer Werte als die Bildgruppe auf und beide zeigten Werte über denen der Versuchsgruppe. Die implizit erhobenen Daten lassen eine stärkere Präferenz des attraktiven Ideals und der «Beauty is Good»-Stereotype, welche besagt, dass attraktiven Personen eher positive und unattraktiven Personen eher negative Eigenschaften zugesprochen werden, vermuten (u.a. Dion, Berscheid, und Walster 
1972; Engeln-Maddox 2006; Feingold 1992). Daraus kann geschlossen werden, dass die Bewegtbild-Sequenz den grössten Einfluss auf die Versuchspersonen hatte. Diese Ergebnisse bestätigen den untersuchten Zusammenhang.

Die Auswertung der Korrelationen zeigte keine signifikanten Zusammenhänge zwischen Fernsehkonsum, Internetkonsum sowie dem Konsum von Printmedien und der Bewertung des eigenen Körpers. Das heisst, ein Zusammenhang zwischen hohem Medienkonsum und einem negativen Körperbild, wie angenommen, konnte nicht festgestellt werden.

Die Jugendlichen gaben in Rückmeldungen an, dass sie permanent dem medial verbreiteten Ideal ausgesetzt sind und sich dadurch nicht oder nicht mehr beeinflussen lassen. Dies würde der Theorie des Kultivationsansatzes widersprechen. Es drängt sich nun die Annahme auf, dass ein häufiger Medienkonsum nicht automatisch zur Verinnerlichung des Ideals führt, sondern gegeben falls eine gewisse Habitualisierung mit sich bringen kann. Die permanente mediale Präsentation des Ideals kann zu einer Desensibilisierung und folglich zu weniger Aufmerksamkeit durch die Rezipientinnen und Rezipienten führen (vgl. Holmstrom 2004). Diese Wirkzusammenhänge werfen eine entscheidende Frage für weiterführende Forschungen auf.

Interessante Befunde lieferte die Auswertung der Figure Rating Scale. Hier sind Unterschiede in der Bewertung der Mädchen und der Jungen ersichtlich. Zusammenfassend kann festgestellt werden, dass Mädchen deutlich unzufriedener bezüglich ihrer eigenen Figur waren (vgl. Schemer 2003). Sie idealisierten für männliche und weibliche Körper eine dünnere Silhouette als die Jungen. Die Diskrepanz zwischen dem weiblichen Wunschkörper und dem von männlichen Jugendlichen bevorzugten Frauenkörper war deutlich sichtbar (vgl. Harrison 2000). Dies gibt Anlass, geschlechtergemischt über Wunschvorstellungen zu sprechen und so die Anspannung und den Druck von den jungen Mädchen zu nehmen. Dieser Punkt wird in der Durchführung einer weiteren Studie berücksichtigt (Baumgartner-Hirscher 2017).

\section{Zusammenfassung der Ergebnisse}

Auf expliziter Ebene konnten keine signifikanten Effekte festgestellt werden. Die Erweiterung auf implizite Methoden zeigte jedoch einen signifikanten Effekt nach der Rezeption medialer Schönheitsideale. Es kann jedoch die Annahme getroffen werden, dass keine sozialen Vergleichsprozesse stattgefunden haben, beziehungsweise diese keine Auswirkungen auf die persönliche Körperzufriedenheit der Versuchspersonen zeigten (vgl. Schemer 2007). Die hohe Körperzufriedenheit und die hohe Selbstakzeptanz des eigenen Körpers der Versuchspersonen unterstützt diese Annahme. Auch die Verinnerlichung der «Beauty is Good»-Stereotype konnte bestätigt werden (vgl. Dion et al. 1972; Griffin und Langlois 2006; Tsukiura und Cabeza 2011). 


\section{Fazit und Ausblick}

Die Zunahme von Körperunzufriedenheiten und die Entwicklung von Essstörungen können nicht ausschliesslich auf den Konsum von Werbung, Filmen, Serien oder anderen Medieninhalten zurückgeführt werden. Festzustellen ist jedoch, dass in Zeiten mit einer derartig ausgedehnten massenmedialen Kommunikation Ideale und der Druck diesen zu entsprechen, schneller und - vor dem Hintergrund wirtschaftlicher Interessen - effizienter verbreitet werden können.

Der soziale Druck, dem medial vermittelten Schönheitsideal zu entsprechen, wird als Einflussfaktor bei der Entstehung von Körperbildstörungen und im Weiteren zur Begünstigung oder Aufrechterhaltung einer Essstörung gesehen (vgl. Polivy und Herman 2002; Warschburger, Krentz, und Helfert 2015).

Um diesen Druck abzuschwächen, thematisieren primärpräventive Massnahmen wie und warum es zur Verbreitung solcher Ideale kommt (Ridolfi und Wander Val 2008). Das Ziel primärpräventiver Massnahmen ist dort anzusetzen, wo noch keine Veränderungen hinsichtlich des zu vermeidbaren Verhaltens auftreten (vgl. Becker 1997). Hier werden verschiedenen Ansätze thematisiert, zum Beispiel die Aufklärung über vorangegangenen Retuscheoperation in den Printmedien oder Wirkmechanismen von Werbung. Primärpräventive Massnahmen bedienen sich dazu unterschiedlicher Methoden. Neben der Vermittlung von Wissen stellt die Stärkung der Medienkompetenz, insbesondere hinsichtlich des kritischen Hinterfragen der vermittelten Ideale und Rollenbilder, einen zentralen Punkt dar (vgl. Berger 2006; Brokemper 2009; Dannigkeit et al. 2005; Dennhardt 2008; Levine und Smolak 1998; Schlevogt 2002).

Wichtig ist es hier, die Kompetenzen der Kinder und Jugendlichen hinsichtlich der Rezeption und weiteren Verarbeitung medialer Inhalte weitgehend zu stärken, Motive und Erwartungen realistisch darzustellen und den Umgang mit neuen Medien als Hilfsmittel im weiteren persönlichen Leben positiv zu gestalten. BaumgartnerHirscher (2017). Derartige Inhalte sollten in der Sekundarstufe I in den Unterricht eingebaut werden. Damit kann gewährleistet werden, dass während der Pflichtschulzeit flächendeckend alle Schülerinnen und Schüler erreicht werden können.

Aufbauend auf die vorliegende Studie wurde auch eine medienpädagogische Kurzintervention für den Unterricht konzipiert. Ziel dieser Intervention war es, möglichen Auswirkungen des Medienkonsums auf das jugendliche Körperbild entgegenzuwirken. Inhalte dieser Unterrichtssequenz sind unter anderem die gemeinsame Erarbeitung und Diskussion des derzeitigen Schönheitsideals für beide Geschlechter und die Analyse von Printwerbungen hinsichtlich Körperbild, Rollenbild und Werbebotschaft. Die Entwicklung dieses Ansatzes soll dazu beitragen, dass Lehrenden gezielt ein praxisnaher medienpädagogischer Ansatz zur Verfügung steht, um etwaigen negativen Auswirkungen medialer Angebote auf das Körperbild von Jugendlichen aktiv und forschungsbasiert entgegenzuwirken. 


\section{Literatur}

Aronson, Elliot, Timothy Wilson, und Robin Akert. 2009. Sozialpsychologie. München: Pears Education.

Banaji, Mahzarin Rustum. 2001. «Implicit attitudes can be measured». In The nature of remembering: Essays in honor of Robert G. Crowder, hrsg. v. Henry L. Roediger, James S. Nairne, Ian Neath u. Aimée M. Surprenant, 117-150. Washington DC: American Psychological Association.

Bandura, Albert. 1971. Social learning theory. New York: General Learning Press.

Baumann, Eva. 2009. Die Symptomatik des Medienhandelns. Zur Rolle der Medien im Kontext der Entstehung, des Verlaufs und der Bewältigung eines gestörten Essverhaltens. Cologne: Herbert von Halem.

Baumgartner-Hirscher, Natalie. 2017. Die Effekte der Darstellung von Attraktivitätsidealen in ausgewählten medialen Angeboten auf das Körperbild von Jugendlichen: eine neue Erziehungsaufgabe für die Biologiedidaktik in der Schule (unveröffentlichte Dissertationsschrift). Salzburg: Universität Salzburg.

Becker, Peter. 1997. «Prävention und Gesundheitsförderung». In Gesundheitspsychologie. Ein Lehrbuch, hrsg. v. Ralf Schwarzer, 517-34. 2. Auflage. Göttingen: Hogrefe.

Berger, Uwe. 2006. «Primärprävention bei Essstörungen». Psychotherapeut 51 (3): 187-196. https://doi.org/10.1007/s00278-006-0489-7.

Borelli, Claudia, und Mirjam Berneburg. 2010. «Schönheit liegt im Auge des Betrachters? Aspekte von Schönheit oder Attraktivität». Journal der Deutschen Dermatologischen Gesellschaft 8 (5): 326-331. https://doi.org/10.1111/j.1610-0387.2009.07318.x.

Braun, Christoph, Martin Gründl, Claus Marberger, und Christoph Scherber. 2001. Beautycheck - Ursachen und Folgen von Attraktivität. Zugriff 2.4.2017. http://www.beautycheck.de/ cmsms/index.php/der-ganze-bericht.

Brokemper, Peter. 2009. Schönheit - ein Projektbuch: Hintergründe - Perspektiven - Denkanstöße. Mühlheim: Verlag an der Ruhr.

Brunner, Romuald, und Franz Resch. 2015. «Diätverhalten und Körperbild im gesellschaftlichen Wandel». In Handbuch Essstörungen und Adipositas, hrsg. v. Stephan Herpertz, Martina De Zwaan u. Stephan Zipfel, 9-14. Heidelberg: Springer.

Cunningham, Michael R. 1986. «Measuring the physical in physical attractiveness: Quasi-experiments on the sociobiology of female beauty». Journal of Personality and Social Psychology 50 (5): 925-935. https://doi.org/10.1037/0022-3514.50.5.925.

Cunningham, Michael R., Alan R. Roberts, Anita P. Barbee, Perri B. Druen, und Cheng-Huan Wu. 1995. «Their ideas of beauty are, on the whole, the same as ours: Consistency and variability in the cross-cultural perception of female physical attractiveness». Journal of Personality and Social Psychology 68 (2): 261-279. https://doi.org/10.1037/0022-3514.68.2.261.

Cunningham, Michael R., Anita P. Barbee, und Correna L. Philhower. 2002. «Dimensions of facial physical attractiveness: The intersection of biology and culture». In Advances in visual cognition. Facial attractiveness: Evolutionary, cognitive, and social perspectives, hrsg. v. Gillian Rhodes u. Leslie A. Zebrowitz, 193-238. Westport: Ablex Publishing. 
Cuntz, Ulrich und Andreas Hillert. 2008. Essstörungen: Ursachen, Symptome, Therapien. München: $\mathrm{CH}$ Beck.

Cusumano, Dale L., und J. Kevin Thompson. 2001. «Media influence and body image in 8-11-Year-old boys and girls: A preliminary report on the multidimensional media influence scale». International Journal of Eating Disorders 29 (1): 37-44. https://doi.org/10.1002/1098108X(200101)29:1<37::AID-EAT6>3.0.CO;2-G.

Dannigkeit, Nora, Grit Köster, und Brunna Tuschen-Caffier. 2007. Prävention von Essstörungen - Ein Trainingsprogramm zum Einsatz an Schulen. Tübingen: DGVT.

Dennhardt, Ulrike. 2008. Prävention von Essstörungen. Präventions- und Therapiemöglichkeiten von Magersucht, Bulimie und Binge-Eating. Saarbrücken: Dr. Müller.

Deusinger, Ingrid. 1998. Die Frankfurter Körperkonzeptskalen (FKKS). Göttingen: Hogrefe.

Dion, Karen, Ellen Berscheid, und Elaine Walster. 1972. «What is beautiful is good». Journal of personality and social psychology 24 (3): 285.

Dittmar, Helga. 2005. «Introduction to the special issue: Body image-vulnerability factors and processes linking sociocultural pressures and body dissatisfaction». Journal of Social and Clinical Psychology 24 (8): 1081-1087. https://doi.org/10.1521/jscp.2005.24.8.1081.

Dittmar, Helga, und Sarah Howard. 2004. «Thin-ideal internalization and social comparison tendency as moderators of media models' impact on women's body-focused anxiety». Journal of Social and Clinical Psychology 23 (6): 768-791. https://doi.org/10.1521/ jscp.23.6.768.54799.

Dolan, Bridget. 1996. «Die Bedeutung soziokultureller Faktoren für die Entwicklung von Essstörungen». In Beschädigte Weiblichkeit - Eßstörungen, Sexualität und sexueller Missbrauch, hrsg. v. Werner Köpp u. Georg E. Jacobi, 30-37. Heidelberg: Roland Asanger.

Engeln-Maddox, Renee. 2005. «Cognitive responses to idealized media images of women: The relationship of social comparison and critical processing to body image disturbance in college women». Journal of Social and Clinical Psychology 24 (8):1114-1138. https://doi. org/10.1521/jscp.2005.24.8.1114.

Engeln-Maddox, Renee. 2006. «Buying a Beauty Standard or Dreaming of a new Life? Expectations associated with Media Ideals». Psychology of Woman Quarterly 30 (3): 258-266. https://doi.org/10.1111/j.1471-6402.2006.00294.x.

Feingold, Alan. 1992. «Good-looking people are not what we think». Psychological Bulletin 111 (2): 304-341.

Festinger, Leon. 1954. «A Theory of Social Comparison Processes». Human Relations 7 (2): 117-140.

Fichter, Manfred M. 2008. «Anorektische und bulimische Eßstörungen». Ernährung-Wissenschaft und Praxis 2 (4): 148-155. https://doi.org/10.1007/s12082-008-0167-y.

Fichter, Manfred. 2013. Magersucht und Bulimia: empirische Untersuchungen zur Epidemiologie, Symptomatologie, Nosologie und zum Verlauf. Band 37. Berlin: Springer.

Garner, David. M. 1991. «Soziokulturelle Aspekte bei Eßstörungen». In Bulimia und Anorexia nervosa, hrsg. v. Corinna Jacobi u. Thomas Paul, 11-23. Berlin u. Heidelberg: Springer. 
Geissner, Edgar, und Katrin Schary. 2005. «Familiale und soziokulturelle Faktoren bei Essstörungssymptomen». Zeitschrift für Gesundheitspsychologie 13 (2): 44-57. https://doi. org/10.1026/0943-8149.13.2.44.

Gerbner, George, Larry Gross, Michael Morgan, und Nancy Signorielli. 1986. «Living with television: The dynamics of the cultivation process». Perspectives on media effects: 17-40.

Gerlinghoff, Monika, und Herbert Backmund. 2004. «Essstörungen im Kindes-und Jugendalter». Bundesgesundheitsblatt-Gesundheitsforschung-Gesundheitsschutz 47 (3): 246-250. https://doi.org/10.1007/s00103-003-0791-2.

Gille, Gisela. 2014. «Körperbild und Essstörungen bei jungen Mädchen». Gynäkologe 47, 293302. Heidelberg: Springer. https://doi.org/10.1007/s00129-014-3334-z.

Grabe, Shelly, L. Monique Ward, und Janet S. Hyde. 2008. «The role of the media in body image concerns among women: a meta-analysis of experimental and correlational studies». Psychological bulletin 134 (3): 476. https://doi.org/10.1037/0033-2909.134.3.460.

Greenwald, Anthony G., Debbie E. McGhee, und Jordan L. Schwartz. 1998. «Measuring individual differences in implicit cognition: the implicit association test». Journal of personality and social psychology 74 (6): 1480.

Greenwald, Anthony G., Andrea T. Poehlman, Eric L. Uhlmann, und Mahzarin R. Banaji. 2009. "Understanding and using the Implicit Association Test: III. Meta-analysis of predictive validity». Journal of personality and social psychology 97 (1): 17. https://doi.org/10.1037/ a0015575.

Griffin, Angela M., und Judith H. Langlois. 2006. «Stereotype directionality and attractiveness stereotyping: Is beauty good or is ugly bad?». Social Cognition 24 (2): 212-246. https://doi. org/10.1521/soco.2006.24.2.187.

Hargreaves, Duane A., und Marika Tiggemann. 2003. "Female "thin ideal" media images and boys' attitudes toward girls». Sex Roles 49 (9-10): 539-544. https://doi. $\operatorname{org} / 10.1023 / A: 1025841008820$.

Hargreaves, Diane A., und Marika Tiggemann. 2004. «Idealized media images and adolescent body image: Comparing boys and girls». Body image 1 (4): 351-361. https://doi. org/10.1016/j.bodyim.2004.10.002.

Harrison, Kristen. 2000. «The body electric:thin-ideal media and eating disordersin adolescents». Journal of Communication 50 (3): 119-143. https://doi.org/10.1111/j.1460-2466.2000. tb02856.x.

Hawkins, Nicole, P. Scott Richards, H. Mac. Granley, und David M. Stein. 2004. «The impact of exposure to the thin-ideal media image on women». Eating disorders 12 (1): 35-50. https:// doi.org/10.1080/10640260490267751.

Heinberg, Leslie J., und J. Kevin Thompson. 1995. «Body image and televised images of thinness and attractiveness: A controlled laboratory investigation». Journal of social and clinical psychology 14 (4): 325-338. https://doi.org/10.1521/jscp.1995.14.4.325.

Hölling, Heike, und Robert Schlack. 2007. «Essstörungen im Kindes- und Jugendalter». Bundesgesundheitsblatt - Gesundheitsforschung - Gesundheitsschutz 50 (5): 794-799. https:// doi.org/10.1007/s00103-007-0242-6. 
Holmstrom, Amanda. 2004. «The effects of the media on body image: A meta-analysis». Journal of Broadcasting \& Electronic Media 48 (2): 196-217. https://doi.org/10.1207/ s15506878jobem4802_3.

Hönn, Mirjam, und Gernot Göz. 2007. «The Ideal of Facial Beauty: A Review». Journal of Orofacial Orthopedics 68 (1): 6-26. https://doi.org/10.1007/s00056-007-0604-6.

Jacobi, Corinna, Thomas Paul, und Andreas Thiel. 2004. Essstörungen. Band 24. Göttingen: Hogrefe.

Jäger, Burkard. 2015. «Soziokulturelle Aspekte der Essstörungen». In Handbuch Essstörungen und Adipositas, hrsg. v. Stephan Herpertz, Martina De Zwaan u. Stephan Zipfel, 133-140. Heidelberg: Springer.

Jonas, Klaus, und Philip Brömer. 2002. «Die sozial-kognitive Theorie von Bandura». In Theorien der Sozialpsychologie. Band II. Gruppen, Interaktions- und Lerntheorien, hrsg. v. Dieter Frey u. Martin Irle, 277-299. Bern: Hans Huber.

Klusmann, Dietrich, und Wolfgang Berner. 2011. «Veränderungen weiblicher Partnerpräferenzen im Menstruationszyklus». Zeitschrift für Sexualforschung 24 (02): 170-186. https://doi. org/10.1055/s-0031-1271481.

Lang, Peter J., Margaret M. Bradley, und Bruce N. Cuthbert. 1999. International affective picture system (IAPS): Instruction manual and affective ratings. The center for research in psychophysiology, Florida: University of Florida.

Langlois, Judith H., und Lori A. Roggman. 1990. «Attractive faces are only average». Psychological Science 1: 115-121. https://doi.org/10.1111/j.1467-9280.1990.tb00079.x.

Leffelsend, Stefanie, Martina Mauch, und Bettina Hannover. (2004). «Mediennutzung und Medienwirkung». In Lehrbuch der Medienpsychologie, hrsg. v. Roland Mangold, Peter Vorderer u. Gary Bente, 51-71. Göttingen: Hogrefe.

Levine, Michael P., und Linda Smolak. 1998. «The mass media and disordered eating: Implications for primary prevention». In The prevention of eating disorders, hrsg. v. Walter Vandereycken u. Great Noordenbos, 23-56. New York: New York University Press.

Lorenz, Konrad. 1943. «Die angeborenen Formen möglicher Erfahrung». Ethology 5 (2): 235409. https://doi.org/10.1111/j.1439-0310.1943.tb00655.x.

McCabe, Marita P., und Lina A. Ricciardelli. 2001. "Parent, peer, and media influences on body image and strategies to both increase and decrease body size among adolescent boys and girls». Adolescence 36 (142): 225.

McCabe, Marita. P., und Lina A. Ricciardelli. 2005. «A prospective study of pressures from parents, peers, and the media on extreme weight change behaviors among adolescent boys and girls». Behaviour research and therapy 43 (5): 653-668. https://doi.org/10.1016/j. brat.2004.05.004.

Niedballa, Christin. 2011. Tanz Dich schön!: Tanzpädagogische Projekte als mögliche Prävention von Essstörungen an weiterführenden Schulen. Saarbrücken: VDM Verlag Dr. Müller.

Paus-Hasebrink, Ingrid, und Michelle Bichler. 2008. Mediensozialisationsforschung. Theoretische Fundierung und Fallbeispiel sozial benachteiligter Kinder. Innsbruck: Studienverlag. 
Perrett, David I., D. Michael Burt, Ian S. Penton-Voak, Kieran J Lee., A. Duncan A. Rowland, und Rachel Edwards. 1999. "Symmetry and human facial attractiveness». Evolution and human behavior 20 (5): 295-307. https://doi.org/10.1016/S1090-5138(99)00014-8.

Pinhas, Leora, Brenda B. Toner, Alisha Ali, Paul E. Garfinkel, und Noreen Stuckless. 1999. "The effects of the ideal of female beauty on mood and body satisfaction». International Journal of Eating Disorders 25 (2): 223-226. https://doi.org/10.1002/(SICI)1098108X(199903)25:2<223::AID-EAT12>3.0.CO;2-B.

Polivy, Janet, und Peter C. Herman. 2002. "Causes of eating disorders». Annual review of psychology: 53 (1): 187-213. https://doi.org/10.1146/annurev.psych.53.100901.135103.

Rhodes, Gillian, Sakiko Yoshikawa, Alison Clark, Kieran Lee, Kieran, Ryan McKay, und Shigeru Akamatsu. 2001. "Attractiveness of facial averageness and symmetry in non-Western cultures: In search of biologically based standards of beauty». Perception 30 (5): 611-625. https://doi.org/10.1068/p3123.

Ridolfi, Danielle R, und Jillion S. Vander Wal. 2008. «Eating disorders awareness week: The effectiveness of a one-time body image dissatisfaction prevention session». Eating disorders 16 (5): 428-443. https://doi.org/10.1080/10640260802370630.

Schemer, Christian. 2003. «Schlank und krank durch Medienschönheiten? Zur Wirkung attraktiver weiblicher Medienakteure auf das Körperbild von Frauen». M\&K Medien \& Kommunikationswissenschaft 51 (3-4): 523-540. https://doi.org/10.5167/uzh-26741.

Schemer, Christian. (2007). «Wem Medienschönheiten schaden: Die differenzielle Anfälligkeit für negative Wirkungen attraktiver Werbemodels auf das Körperbild junger Frauen». Zeitschrift für Medienpsychologie 19 (2): 58-67. https://doi.org/10.1026/1617-6383.19.2.5.

Schlevogt, Vanessa. 2002. Präventionsprojekt „Jugend mit Biss“. Frankfurt: Selbstverlag Frankfurter Zentrum für Ess-Störungen.

Schorb, Bernd. 2005. «Sozialisation». In Grundbegriffe der Medienpädagogik, hrsg. v. Jürgen Hüther u. Bernd Schorb, 382-389. München: KoPäd.

Schramm, Holga, und Uwe Hasebrink. 2004. «Fernsehnutzung und Fernsehwirkung». In Lehrbuch der Medienpsychologie, hrsg. v. Roland Mangold, Peter Vorderer u. Gary Bente, 465492. Göttingen: Hogrefe.

Schuster, Michael. 1993. "Gesichtsschönheit: Begriff, Geschichte und Merkmale». In Physische Attraktivität, hrsg v. Manfred Hassebrauck u. Reiner Niketta, 11-28. Göttingen: Hogrefe.

Slater, Amy, und Marika Tiggemann. 2006. «The contribution of physical activity and media use during childhood and adolescence to adult women's body image». Journal of health psychology 11 (4): 553-565. https://doi.org/10.1177/1359105306065016.

Šmahel, David, Hana Macháčková, Martina Šmahelová, Michal Čevelíček, Carlos Almenara, und Jana Holubčíková. (2018). Digital Technology, Eating Behaviors, and Eating Disorders. Springer.

Smolak, Linda, Michael P. Levine, und Florence Schermer. 1998. «A controlled evaluation of an elementary school primary prevention program for eating problems». Journal of Psychosomatic Research 44 (3): 339-353. https://doi.org/10.1016/S0022-3999(97)00259-6. 
Stahr, Ingeborg, Ingrid Barb-Priebe und Elke Schulz. 2003. Essstörungen und die Suche nach Identität. Weinheim: Juventa.

Stice, Eric, und Sarah K. Bearman. 2001. «Body-image and eating disturbances prospectively predict increases in depressive symptoms in adolescent girls: a growth curve analysis». Developmental psychology 37 (5): 597. https://doi.org/10.1037/0012-1649.37.5.597.

Stice, Eric, Erika Schupak-Neuberg, Heather E. Shaw, und Richard Stein. 1994. «Relation of media exposure to eating disorder symptomatology: An examination of mediating mechanism». Journal of Abnormal Psychology 103: 836-844. https://doi.org/10.1037/0021843x.103.4.836.

Stunkard, Albert J., T. Sorensen, und Fini Schulsinger. 1983. Use of the Danish Adoption Register for the study of obesity and thinness. The genetics of neurological and psychiatric disorders. New York: Raven, 115-120.

Thornton, Bill, und Scott Moore. 1993. «Physical Attractiveness Contrast Effect: Implications for Self-Esteem and Evaluations of the Social Self». Personality and Social Psychology Bulletin 19: 474-480. https://doi.org/10.1177/0146167293194012.

Treumann, Klaus P., Dorothee M. Meister, Uwe Sander, Eckard Burkatzki, Jörg Hagedorn, Manuela Kämmerer, Mareike Strotmann, und Claudia Wegener. 2007. Medienhandeln Jugendlicher: Mediennutzung und Medienkompetenz. Bielefelder Medienkompetenzmodell. Berlin: Springer.

Tsukiura, Takashi, und Roberto Cabeza. 2011. «Shared brain activity for aesthetic and moral judgments: implications for the Beauty-is-Good stereotype». Social Cognitive and Affective Neuroscience 6 (1): 138-148. https://doi.org/10.1093/scan/nsq025.

Van Bebber, Kira. 2013. «Neue TV-Formate im pädagogischen Blick». Zeitschrift für Erziehungswissenschaft 16 (4): 799-803. https://doi.org/10.1007/s11618-013-0445-6.

Vocks, Silja, und Tanja Legenbauer. 2010. Körperbildtherapie bei Anorexia und Bulimia nervosa: ein kognitiv-verhaltenstherapeutisches Behandlungsprogramm. Göttingen: Hogrefe.

Warschburger, Petra, Eva M. Krentz, und Susanne Helfert. 2015. «Soziokulturelle Faktoren und Essstörungen im Jugendalter: Evaluation eines schulbasierten Präventionsprogramms». Das Gesundheitswesen 77 (1): 58-59. https://doi.org/10.1055/s-0032-1330023.

Wegener, Claudia. 2008. Medien, Aneignung und Identität.,Stars“ im Alltag jugendlicher Fans. Wiesbaden: Verlag für Sozialwissenschaften.

Yamamiya, Yuko, Thomas F. Cash, Susan E. Melnyk, Heidi D. Posavac, und Steven S. Posavac. 2005. «Women's exposure to thin-and-beautiful media images: Body image effects of media-ideal internalization and impact-reduction interventions». Body image 2 (1): 74-80. https://doi.org/10.1016/j.bodyim.2004.11.001.

Zajonc, Robert B. 1968. «Attitudinal effects of mere exposure». Journal of personality and social psychology 9 (2p2):1. https://doi.org/10.1037/h0025848. 